\title{
Gastrointestinal stromal tumors: A clinical and histopathological presentation of 27 cases
}

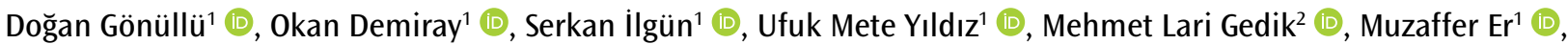

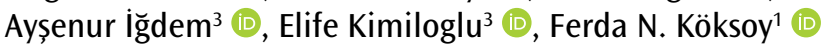

ABSTRACT

ORCID IDs of the authors: D.G. 0000-0002-8232-5209; 0.D. 0000-0001-6920-7585; S.I. 0000-0002-4862-2891; U.M.Y. 0000-0002-4482-0225; M.L.G. 0000-0002-0440-9394; M.E. 0000-0001-8578-1229; A.I. 0000-0002-3047-1546; E.K. 0000-0002-2708-1272; F.N.K. 0000-0002-5878-7489.

\section{Cite this paper as:} Gönüllü D, Demiray 0, illgün S, Yıldız UM, Gedik ML, Er $M$, et al. Gastrointestinal stromal tumors: A clinical and histopathological presentation of 27 cases. Turk J Surg 2018; 34(4): 259-263.

'Department of General Surgery, University of Health Sciences, Gaziosmanpaşa Taksim Training and Research Hospital, İstanbul, Turkey

2Department of General Surgery, Ministry of Health, Fatsa State

Hospital, Ordu, Turkey

${ }^{3}$ Department of Pathology, University of Health Sciences, Gaziosmanpaşa Taksim Training and Research Hospital, İstanbul, Turkey This study was presented at the $36^{\text {th }}$ Congress of the European Society of Surgical Oncology, 14-16 September 2016, Krakow, Poland.

\section{Corresponding Author} Doğan Gönüllï e-mail: dogangonullu@yahoo.com

Received: 08.11.2017 Accepted: 07.02.2018

Available Online Date: 11.09 .2018

(c) Copyright 2018 by Turkish Surgical Association Available online at turkjsurg.com objective: Gastrointestinal stromal tumors (GISTs) are mesenchymal tumors that express type 3 tyrosine kinase receptors and are thought to develop from the neoplastic transformation of the interstitial Cajal cells. The present study was performed to morphologically and immunohistologically evaluate GISTs, to compare their qualities using a GIST risk categorization system, and to identify the diagnostic and prognostic parameters of GISTs.

Material and Methods: A total of 27 patients with GISTs underwent treatment and were followed up at the Gaziosmanpaşa Taksim Training and Research Hospital. Descriptive statistics was used to calculate the mean and median values. Survival analysis was performed by the Kaplan-Meier method. The analyses were performed using the SPSS version 22.0 software.

Results: The mean follow-up time was 3.5 (5 months to 13 years) years. The mean age was 60.4 (29-82) years. The tumors were localized in the stomach (62.9\%), extraintestinal areas (14.8\%), intestine (7.4\%), esophagus (7.4\%), and rectum (7.4\%). Twenty-four patients were classified according to the Fletcher system. Of these patients, 7 (25.9\%) were classified as very low risk, 8 (29.6\%) as low risk, $7(25.9 \%)$ as intermediate risk, and $2(7.4 \%)$ as high risk. Twenty-four patients underwent surgery. Of the 3 patients who did not undergo surgery, 1 had metastatic disease at the time of diagnosis, and 2 had mini- or micro-GISTs in the stomach. On endoscopic surveillance, all tumors remained stable. Three out of the 27 patients were lost to follow-up. Two patients developed recurrence, and 1 patient died of GIST.

Conclusion: We analyzed the clinical and pathological characteristics of GIST. The most common site of tumor origin was the stomach. The size, mitotic index, and Ki-67 values were to be found high in intermediate- and high-risk groups and metastatic diseases.

Keywords: Gastrointestinal stromal tumor, interstitial Cajal cells, mesenchymal tumors, tyrosine kinase receptor mutation

\section{INTRODUCTION}

Gastrointestinal stromal tumors (GISTs) are mesenchymal tumors that express type 3 tyrosine kinase receptors and are thought to develop from the neoplastic transformation of the interstitial Cajal cells (intestinal pacemaker cells on the intestinal wall) or their precursors (1-3). Mutation of the tyrosine kinase receptor (c-Kit/CD117) in precursor tumors causes uncontrolled growth. Approximately $95 \%$ of sporadic GISTs are positive for CD117,60\% to $70 \%$ positive for CD34, and $30 \%$ to $40 \%$ positive for smooth muscle actin, whereas negative for $\mathrm{S} 100$ protein and desmin $(3,4)$. KIT gene mutation is specific to gastric stromal tumors in the intramembranous component of the tyrosine kinase receptor (exon 11) and in the extramembranous component (exon 9) as well as to tumors located in the intestines and colon, which reportedly have a worse prognosis $(3,5-7)$. Patients with exon 11 mutation $(63.0 \%-83.5 \%)$ have been shown to have better responses to imatinib (Gleevec; Novartis Pharma AG, Basel, Switzerland) than patients with exon 9 mutation $(34.0 \%-47.8 \%)(3,8,9)$. In patients without KIT mutation, platelet-derived growth factor receptor-alpha (PDGFRa) mutation should be considered, and these types of stromal tumors frequently have epithelioid qualities $(5,10)$. GISTs positive for both KIT and PDGFRa mutations have not been reported; however, tumors that are negative for both markers reportedly constitute approximately $10 \%$ of all GISTs and are predominantly located in the stomach $(2,5,9)$. A highly specific marker, DOG1 (discovered on GIST-1), has been started to be used for c-Kit-negative stromal tumors (2).

The purpose of the present study was to morphologically and immunohistochemically evaluate GISTs, to compare their qualities using the GIST risk categorization table established by Fletcher et al. (11), and to determine the important parameters for the diagnosis and prognosis of GISTs.

\section{MATERIAL AND METHODS}

In total, 27 patients with GISTs underwent surgical treatment and were followed up at the Gaziosmanpaşa Taksim Training and Research Hospital from 2002 to 2016. The study was conducted in accordance with the ethical standards set by the Declaration of Helsinki. Informed consent was obtained from all individual 


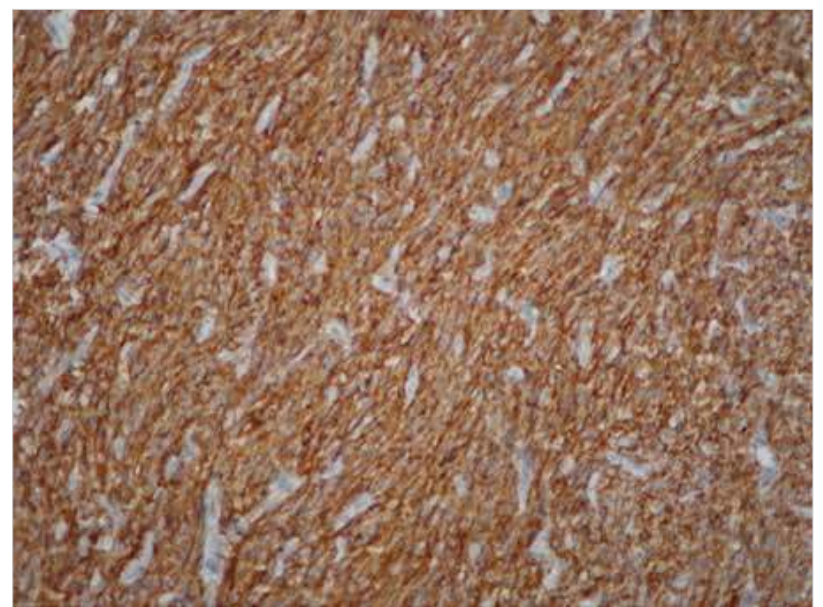

Figure 1. CD117 staining of a gastrointestinal stromal tumor ( $\times 200$ magnification)

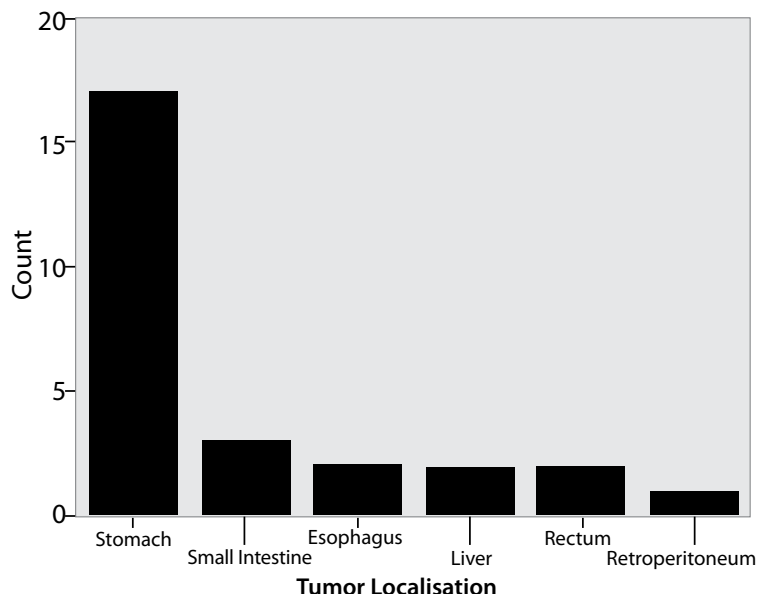

Figure 2. Locations of gastrointestinal stromal tumors

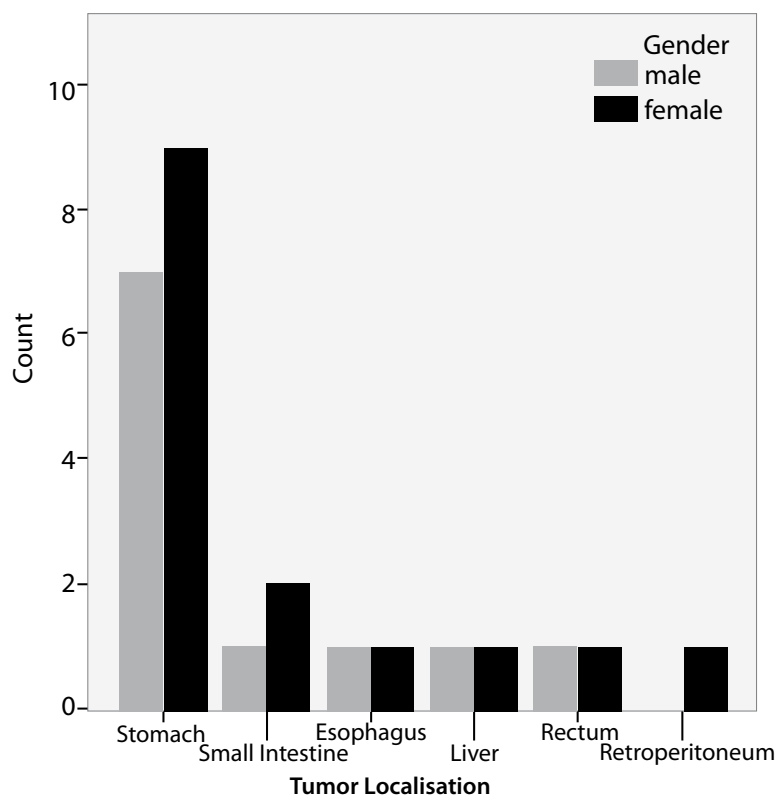

Figure 3. Risk groups according to the Fletcher classification

participants included in the study. Hematoxylin and eosin staining and immunohistochemical markers, such as c-Kit, CD34, and Ki-67, were used to diagnose GISTs (Figure 1). Risk groups and other prognostic parameters were reported (Figure 1). On clini- cal presentation, the median follow-up time was 3.5 (5 months to 13 years) years. Patients in the high-risk group were monitored using an abdominal ultrasonography and a computed tomography every 3 to 6 months for the first 3 years and every 6 months for the following 5 years. Descriptive statistics was used to calculate the mean and median values. Survival analysis was performed by the Kaplan-Meier method. The analyses were performed using the Statistical Package for the Social Sciences (SPSS) version 22.0 software (IBM Corp., Armonk, NY, USA).

\section{RESULTS}

The female-to-male ratio was 5:4. The mean age was 60.4 (29-82) years. Five tumors were incidentally diagnosed in patients undergoing surgery for other reasons, such as gastric cancer, sigmoid volvulus cancer, colon cancer, renal cancer, and ovarian cancer. Seventeen $(62.9 \%)$ tumors were localized in the stomach, 4 (14.8\%) in the extraintestinal areas ( 2 in the mesentery and 2 in the liver), $2(7.4 \%)$ in the intestine, $2(7.4 \%)$ in the esophagus, and 2 (7.4\%) in the rectum (Figure 2 ). Twenty-four patients were classified according to the Fletcher system (3 patients were classified as stage 4). Of all 27 patients, 7 (25.9\%) were classified as very low risk, 8 (29.6\%) as low risk, 7 (25.9\%) as intermediate risk, and 2 (7.4\%) as high risk (Figure 3).

The mean Ki-67 of the patients with stage 4 cancer was 15.6 (725), whereas it was 9.6 (2-25) for both intermediate and highrisk patients. The Ki- 67 level was $>7 \%$ in all patients with metastatic GISTs but in only 6 (54\%) of the intermediate and high-risk patients. The Ki-67 staining ratio was $<1 \%$ for 7 (25.9\%) patients, $2 \%-7 \%$ for $13(48.1 \%)$ patients, and $>7 \%$ for 7 (25.9\%) patients. Staining was not observed in $>7 \%$ of the tumors located in the esophagus; however, staining $>7 \%$ was observed in only 1 patient among those with intestinal tumors (Figure 4).

The mean tumor diameter was $5.45(0.5-18) \mathrm{cm}$, and the largest tumors were observed among those with an extraintestinal localization (average tumor diameter: $13.2(8-18) \mathrm{cm}$ ) (Figure 5). Seven tumors were $<2 \mathrm{~cm}, 3$ were classified as micro-GISTs $(<1 \mathrm{~cm})$, and 4 were classified as mini-GISTs $(1-2 \mathrm{~cm})$.

Fifteen (55.5\%) tumors were spindle cell tumors, 7 (25.9\%) were epithelioid tumors, and 5 (18.5\%) were mixed form tumors (Figure 6). Nine of the 17 GISTs located in the stomach were spindle cell tumors. All esophageal tumors were epithelioid tumors, and tumors localized in the colon or extraintestinal regions were either spindle cell tumors (75\%) or epithelioid tumors (25\%).

Three out of the 27 patients were lost to follow-up. Two patients developed recurrent disease, and one died of GIST. The 5-year survival rate was $89.3 \%$ (Figure 7). Twenty-four (88.8\%) patients underwent surgery. Of the 3 patients who did not undergo surgery, 1 had metastatic disease at the time of diagnosis, and 2 had a mini- or micro-GIST localized in the stomach. On endoscopic surveillance, the tumors remained stable. All patients with high-risk disease and metastatic disease underwent imatinib treatment.

\section{DISCUSSION}

Gastrointestinal stromal tumors are rare and constitute $<1 \%$ of the primary tumors of the gastrointestinal system, $2.2 \%$ of the stomach tumors, $13.9 \%$ of the intestinal tumors, and $0.1 \%$ of the colorectal tumors (12). GISTs are most frequently located in the stomach $(50 \%-60 \%)$, intestine $(20 \%-30 \%)$, colon and rec- 


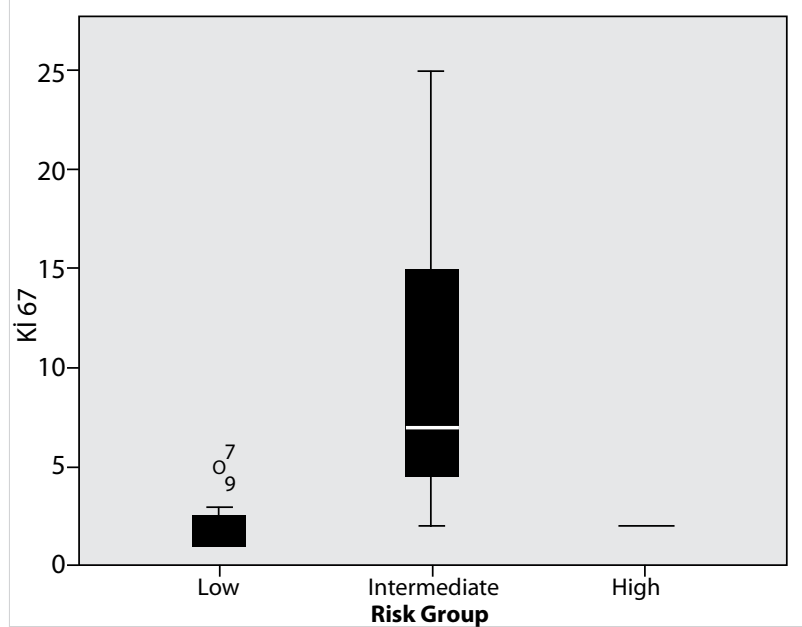

Figure 4. Correlations between $\mathrm{Ki}-67$ index and risk group

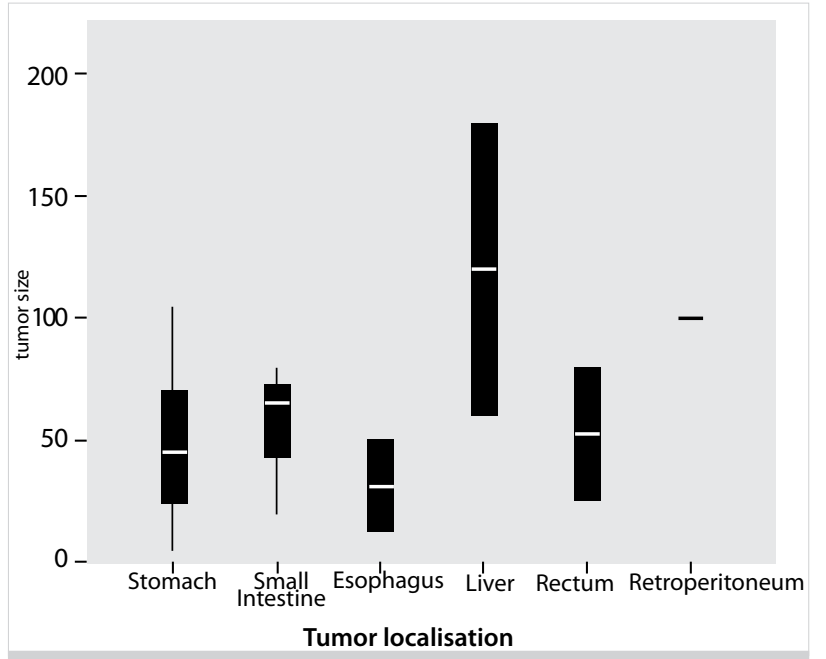

Figure 5. The mean size of gastrointestinal stromal tumors

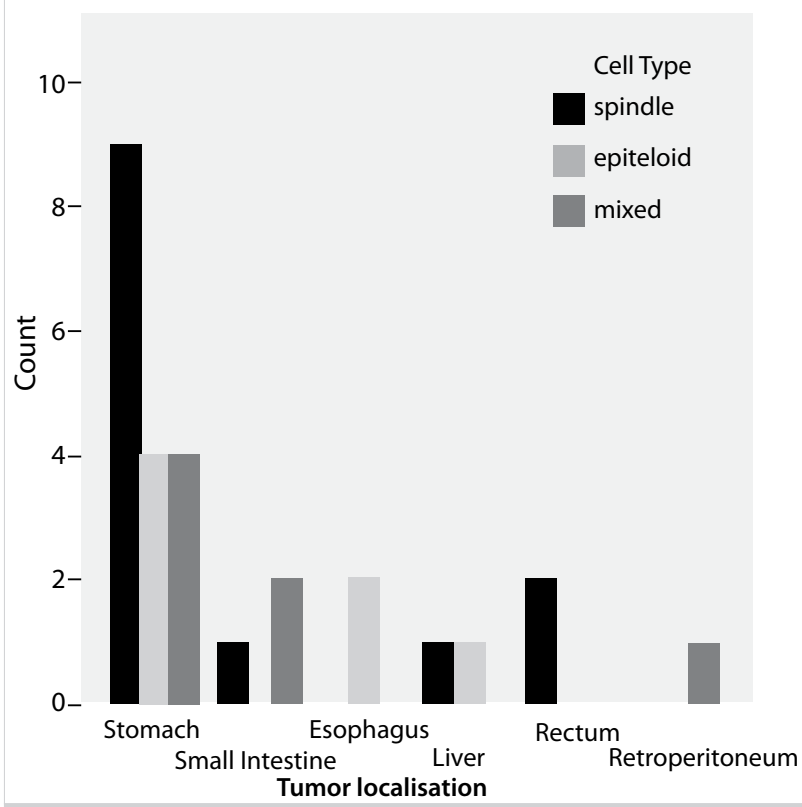

Figure 6. Histological types of gastrointestinal stromal tumors

tum (10\%), and esophagus (5\%). They are rarely located in the extraintestinal regions, such as the mesentery, omentum, and retroperitoneum (5\%) (13). GISTs in the bladder and gallblad-

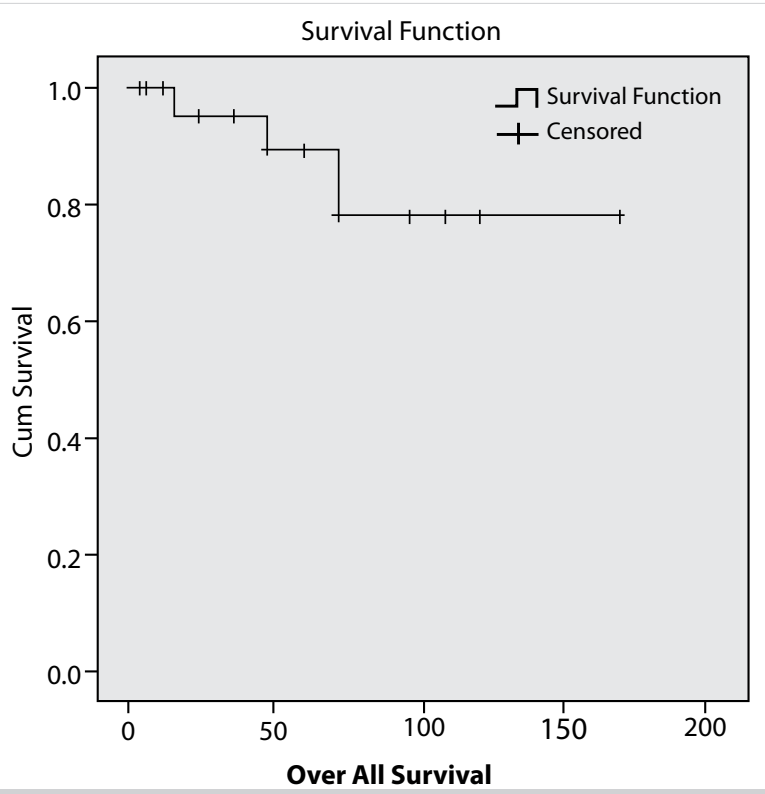

Figure 7. The 5-year survival rate

der have only been described in case reports (14). Consistent with the literature, most GISTs in our series were localized in the stomach (62\%), yet our high ratio of extraintestinal GISTs (14.8\%, 4 liver and retroperitoneal tumors) is a different finding.

Gastrointestinal stromal tumors are equally observed in both sexes. They are more frequent between aged 50 and 70 years. However, the development of GISTs in pediatric patients has also been reported (9). The incidence of GISTs in a Swedish study was 13 per million (15). In other studies, the annual incidence in North America was 3.2 to 7.0 per million, whereas that in Hong Kong was 15 to 20 per million (2). Most GISTs $(80 \%)$ are found incidentally; only a small ratio is observed as a component of a familial disease (e.g., neurofibromatosis type 1 and Carney complex) (9).

Gastrointestinal stromal tumors were historically classified as soft tissue sarcomas (e.g., leiomyosarcoma, leiomyoma, and leiomyoblastoma) owing to their CD34 staining, but the cKit mutation was demonstrated in 1990, after which the term "GIST" began to be used more frequently as a tumor group separate from leiomyosarcoma, schwannoma, and neurofibroma, all of which are c-Kit-negative (4). The Armed Forces Institute for Pathology reviewed 1765 patients who were diagnosed with smooth muscle tumors from 1979 to 1996 and classified $94 \%$ of them as GISTs $(2,3)$.

Histologically, GISTs are classified as spindle cell tumors (70\%), epithelioid cell tumors (20\%), and mixed-type tumors, which are rarely observed. Spindle cell tumors are large in volume, most frequently localized in the stomach and intestines, exhibit KIT and PDGFRa mutations, and respond well to treatment with imatinib mesylate. In contrast, the epithelioid subtype is less frequently observed, does not exhibit KIT and PDGFRa mutations, and co-occurs with lymph node metastases. Spindle cell tumors were observed in $55.5 \%(n=15)$ of the patients, epithelioid in $25.9 \%(n=7)$ of the patients, and mixed in $18.5 \%$ $(n=5)$ of the patients in the present study. Nine of the 17 GISTs were spindle cell tumors, whereas all esophageal tumors were epithelioid. Of those located in the colon or extraintestinally, 
$75 \%$ were spindle cell tumors, and $25 \%$ were epithelioid tumors. In contrast to previous studies, mixed tumors had larger volumes in the present study (average diameter: $6.8 \mathrm{~cm}$ ). c-Kit and CD34 were positive in $86.6 \%$ of the spindle cell tumors, and CD34 was negative in 2 tumors. Additionally, $54.1 \%$ of the epithelioid tumors were positive for both c-Kit and CD34. One case was c-Kit-negative, and the other case was CD34-negative. Among the mixed tumors, c-Kit/CD34 staining was positive in $80 \%$ of the cases, whereas 1 tumor was CD34-negative.

The prognosis and prognostic factors of GISTs have long been the subject of debates. Many parameters have been discussed, but four risk groups were recently accepted based on the tumor size and mitotic index (11). In addition to these two parameters, mucosal invasion, presence of tumor necrosis, increased cellularity, and immunohistochemical markers of cell proliferation (Ki-67, MIB-I, PCNA, and DOG) have been considered as other potentially effective factors in the evaluation of malignancy risk.

The Ki-67 proliferative index is not included among the accepted prognostic factors, yet many studies $(15,16)$ have revealed that it is more valuable than the mitotic index. Nilsson et al. (16) found that in patients with a Ki-67 index $>7$, GIST recurrence is observed in $63 \%$, and $47 \%$ die of this tumor. On the other hand, in patients with a Ki-67 index $<7$, the recurrence rate was only $4 \%$, and only $1 \%$ died of the tumor. Zhao et al. (15) suggested that patients with a Ki- 67 index $>8$ respond less to treatment with tyrosine kinase inhibitors. In our series, the $\mathrm{Ki}-67$ index was $>7 \%$ in 7 patients who were classified as low risk $(n=1)$, intermediate risk $(n=3)$, high risk $(n=1)$, and phase 4 $(n=2)$ based on the Fletcher stratification.

Extraintestinal GISTs develop from multipotent stem cells, which are the precursors of the interstitial Cajal cells. Immunohistochemical studies have shown that they are CD117positive, and according to a previous study, they constitute $10 \%$ of all GISTs (17). In the 2012 San Francisco Gastrointestinal Cancers Symposium, Guye et al. (18) reported that based on the Surveillance, Epidemiology and End-Results records, 261 out of the 2951 patients who underwent surgery from 1996 to 2008 had extraintestinal GISTs with a higher average diameter and were in advanced stages. The 5-year survival rate was 32\% for extraintestinal GISTs and 49\% for GISTs (18). Their report suggested that extraintestinal localization is an independent factor for a poor prognosis. In our series, 4 patients had extraintestinal tumors, 3 had spindle cell tumors, and 1 had an epithelioid stromal tumor. All cases were CD34- and CD117-positive, with an average diameter of $13.2(8-18) \mathrm{cm}$. Two had necrosis, and the Ki-67 ratio was $14.8 \%$ ( $2 \%-25 \%)$. Two were localized in the liver (one was intermediate risk and the other was stage 4 with lung and adrenal metastases). Three were localized in the retroperitoneum or mesentery (intermediate and high risk). Recurrence was observed after 1 year in 1 patient with an intermediate-risk tumor. These patients were followed up for an average of 4 (1-6) years, and all were alive at the time of the present study.

Although GISTs reach a larger volume than other gastrointestinal tumors with adenocarcinoma qualities, they do not lead to lymphatic metastasis, making surgical procedures easier and the prognosis more favorable (8). Their biological behavior varies; only $10 \%$ to $30 \%$ of all GISTs progress to malignancy (13). There is still a lack of consensus on prognostic factors, but the risk clas- sification described by Fletcher et al. (11) is an accepted guideline (18). Tumors displaying malignant behaviors are assessed by tumor size and the mitotic index per 50 high power field (HPF). When the tumor diameter alone is evaluated, a diameter $<5 \mathrm{~cm}$ generally indicates a good prognosis, whereas a diameter $>5 \mathrm{~cm}$ signifies a malignant prognosis. A mitotic index $>5 / 50$ HPF suggests malignancy. However, these parameters have been shown to vary according to localization, and Miettinen et al. (19) of the Armed Forces Institute of Pathology added the GIST localization to the parameters indicated by Fletcher et al. (11). In our series, the use of the Fletcher classification revealed that $25.9 \%(7 / 27)$ of the cases were very low risk, $29.0 \%$ (8/27) were low risk, $25.9 \%$ $(7 / 27)$ were intermediate risk, and $7.4 \%(2 / 27)$ were high risk.

The Memorial Sloan-Kettering Cancer Clinic has developed prognostic nomograms for calculating the 2-year and 5-year recurrence-free rates by evaluating the tumor diameter, mitotic index, and localization findings $(2,3)$.

The fundamental treatment method for most GISTs is surgical resection with clear surgical margins. The extreme fragility of the tumor requires special attention to prevent its rupture $(8,13)$ during the procedure with resultant intra-abdominal seeding. If the tumor ruptures, and if there is no indication for the tyrosine kinase inhibitor imatinib, then it should be noted as an epicrisis and brought to the oncologist's attention; such cases are considered for imatinib treatment regardless of the Fletcher classification (8). Since the possibility of lymphatic metastasis is low in patients with sporadic GISTs $(8,13)$, lymphadenectomy is not necessary $(2,8,13,20,21)$. However, case reports have indicated that the rate of lymph node metastasis is higher in pediatric patients with GISTs that are c-Kit- and PDGFRa-negative, multifocal, or familial (22-24).

Laparoscopic procedures are not recommended for especially large tumors (8). Radiotherapy is ineffective (9). After surgical treatment, recurrence is observed mostly in the peritoneum $(50 \%)$, liver (65\%), or both (13). A review of the previous study suggests that omental tumors recur frequently, and this may be due to rupture and unseen implants. De Matteo et al. (25) reported that among their patients who underwent $\mathrm{R} 0$ resection, $35 \%$ have local recurrence, and $44 \%$ had local recurrence with liver metastases. The tumor diameter is more important than the surgical margin negativity for local recurrence according to their results. We observed recurrence in 2 of the patients. In the first patient, a Fletcher low-risk epithelioid tumor in the stomach fundus was treated by proximal gastrectomy, and recurrence developed at the resection line 4 years later despite the fact that the surgical margin was negative. In the second patient who was determined to have a retroperitoneal spindle cell tumor with intermediate risk, recurrence developed 1 year later and displayed high-risk tumor qualities. Interestingly, two neuroendocrine tumors, each with a diameter of $1 \mathrm{~cm}$, were incidentally detected in the intestine of the second patient.

The 5 -year overall survival rate was $63 \%$ for patients with lowrisk tumors $(<5 \mathrm{~cm})$ and $34 \%$ for those with high-risk tumors $(>10 \mathrm{~cm})$ in the present study $(26)$.

Patients with high-risk tumors ( $>5$ mitoses/50 HPF, tumor diameter $>5 \mathrm{~cm}$, tumor rupture during surgery, or R1-type resection and exon 11 mutation) are considered for adjuvant treat- 
ment (8). In the present series, 5 out of the 27 patients had high-risk stage 4 GISTs according to the Fletcher classification and received imatinib treatment.

We were able to surgically treat $88.8 \%(24 / 27)$ of the patients, and the 5 -year survival rate was $89.3 \%$. Most of the tumors were resectable at the point of diagnosis. Most of the tumors (62.9\%) were located in the stomach, and $81.4 \%(22 / 27)$ of the patients were classified as very low, low, or intermediate risk based on the Fletcher criteria. All of these factors can explain the high survival rate. De Matteo et al. (25) reported a 54\% survival rate and $45 \%$ 5-year remission rate following radical surgery (R0).

\section{CONCLUSION}

We analyzed the clinical and pathological characteristics of GIST admitted and treated during the past 13 years. The most common site of tumor origin was the stomach. The size, mitotic index, and $\mathrm{Ki}-67$ values were found to be high in intermediate- and high-risk groups and metastatic diseases.

Ethics Committee Approval: Authors declared that the research was conducted according to the principles of the World Medical Association Declaration of Helsinki "Ethical Principles for Medical Research Involving Human Subjects" (amended in October 2013).

Informed Consent: Written informed consent was obtained from patients who participated in this study.

Peer-review: Externally peer-reviewed.

Author Contributions: Concept - D.G., O.D., S.I.; Design - D.G., O.D., A.I., E.K.; Supervision - M.E., F.N.K., M.L.G.; Resource - D.G., O.D., E.K.; Materials - D.G., A.I., E.K., M.L.G.; Data Collection and/or Processing F.N.K., S.I., M.L.G., O.D.; Analysis and/or Interpretation - D.G., F.N.K., S.I.; Literature Search D.G., O.D., F.N.K., A.I.; Writing Manuscript - D.G., O.D., S.I.; Critical Reviews - M.E., A.I.., E.K.

Conflict of Interest: The authors have no conflicts of interest to declare.

Financial Disclosure: The authors declared that this study has received no financial support.

\section{REFERENCES}

1. Pidhoreeky I, Chency RT, Kraybill WG, Gibbs JF. Gastrointestinal stromal tumors:current diagnosis, biologic behavior, and management. Ann Surg Oncol 2000: 7; 705-712. [CrossRef]

2. Valsangkar N, Sehdev A, Misra S, Zimmers TA, O'Neil BH, Koniaris LG. Current management of gastrointestinal stromal tumors: Surgery, current biomarkers, mutations, and therapy. Surgery 2015; 158: 1149-1164. [CrossRef]

3. Lai EC, Lau SH, Lau WY. Current management of gastrointestinal stromal tumors-A comprehensive review. Int J Surg 2012; 10: 334-340. [CrossRef]

4. Fletcher CD, Berman JJ, Corless C, Gorstein F, Lasota J, Longley BJ, et al. Diagnosis of Gastrointestinal Stromal Tumors: A consensus Approach. Human Pathology 2002; 33: 459-465. [CrossRef]

5. Neeta Somaiah. Gastrointestinal stromal tumors: optimizing therapy throughout the course of disease. Available from URL: www.clinicaloptions.com/oncology. 2015

6. Lasota J, Corless CL, Heinrich MC, Debiec-Rychter M, Sciot R, Wardelmann E, et al. Maria Debiec-Rychter, Sciot R, Eva W. Clinicopathologic profile of gastrointestinal stromal tumors (GISTs) with primary KIT exon 13 or exon 17 mutations: a multicenter study on 54 cases. Mod Pathol 2008; 21: 476-484. [CrossRef]
7. Taniguchi $M$, Nishida $T$, Hirota $S$, Isozaki $K$, Ito $T$, Nomura $T$, et al. Effect of c-kit Mutation on Prognosis of Gastrointestinal Stromal Tumors. Cancer Res 1999; 59: 4297-4300.

8. Anna Nasierowska-Guttmejer. Gastrointestinal stromal tumors. In Rutkowski P, ed. Soft Tissue Sarcomas. Copyright Via Media: 2016, p: 126-52.

9. Gabriella A. Expression profiling of gastrointestinal stromal tumors biomarkers for prognosis and therapy (MSD Thesis). University of Gothenburg; 2012.

10. Local treatment for gastrointestinal stromal tumors. Available from URL: www.up to date.com

11. Fletcher CD, Berman JJ, Corless C, Gorstein F, Lasota J, Longley BJ, et al. Diagnosis of gastrointestinal stromal tumors: a consensus approach. Int J Surg Pathol 2002: 10: 81-89. [CrossRef]

12. Thomas RM, SobinLH. Gastrointestinal cancer incidence and prognosis by histologic type, SEER population based data 19731983. Cancer 1995: 75; 154-170. [CrossRef]

13. Michael $S$, Emmanouel $D$, Charikleia $S$, Panagiotis $S$, Electra $P$, Georgia L, Michael S. Gastrointestinal stromal tumor. W J of Surg Oncol 2009; 7: 61. [CrossRef]

14. Ortiz-Hidalgo C, de Leon Bojorge B, Albores Saavedra J. Stromal tumor of the gallblader with phenotype of interstitial cells of Cajal. A previous unrecognized neoplasm. Am J Surg Pathol 2000: 24: 1420-1423. [CrossRef]

15. Zhao WY, Xu J, Wang M, Zhang ZZ, Tu L, Wang CJ, et al. Prognostic value of Ki 67 index in gastrointestinal stromal tumors. Int J Clin Exp Pathol 2014; 7: 2298-2304.

16. Nilsson B, Bümming $P$, Meis-Kindblom JM, Oden A, Dortok $A$, Gustavsson B, et al. Gastrointestinal stromal tumors the incidence, prevalence, clinical course, and prognostication in the prematinibmesylat era, a population based study in western Sweden. Cancer 2005; 15; 103; 821-829. [CrossRef]

17. Xiao-Li Luo, Dan Liu, Jian-Jun Yang, Min-Wen Zheng, Jing Zhang, XiaoDong Zhou. Primary gastrointestinal stromal tumor of the liver: A case report. World J Gastroenterol 2009; 7; 15: 3704-3707. [CrossRef]

18. Guye ML. Extraintestinal location for primary non-metastatic GIST associated with increased risk for death. 2012 Gastrointestinal Cancers Symposium. San Fransisco.

19. Miettinen M, Leslie H. Sobin, Maarit Sarlomo-Rikala. Immunohistochemical Spectrum of GISTs at Different Sites and Their Differential Diagnosis with a Reference to CD117 (KIT). Mod Pathol 2000; 13: 1134-1142. [CrossRef]

20. Fletcher CD. Risk aggressive behavior in GIST's. Human Pathology 2002; 33: 459-65. [CrossRef]

21. D'Amato G, Steinert DM, McAuliffe JC, Trent JC. Update on biology and therapy of gastrointestinal stromal tumours. Cancer Control 2005; 12: 44-56. [CrossRef]

22. Shafizad A, Mohammadianpanah M, Nasrolahi H, Mokhtari $M$, Mousavi SA. Lymph Node Metastasis in Gastrointestinal Stromal Tumor (GIST): to Report a Case. Iranian J of Cancer Prevention 2014: 7: 171-175.

23. Otto C, Agaimy A, Braun A, Rädecke J, Hoeppner J, Illerhaus G, et al. Multifocal gastric gastrointestinal stromal tumors (GISTs) with lymph node metastases in children and young adults: A comparative clinical and histomorphological study of three cases includinga new case of Carney triad. Diagn Pathol 2011; 6: 52. [CrossRef]

24. Agaimy $A$, WünschPH. Lymph node metastasis in gastrointestinal stromal tumours (GIST) occurs preferentially in young patients $<$ or $=40$ years: An overview based on our case material and the literature. Langenbecks Arch Surg 2009: 394: 375-381. [CrossRef]

25. DeMatteo RP, Lewis JJ, Leung D, Mudan SS, James M W, Brennan MF. Two Hundred Gastrointestinal Stromal Tumors Recurrence Patterns and Prognostic Factors for Survival. Ann Surg 2000: 231: 51-58. [CrossRef]

26. Besana-Ciani I, Boni L, Dionigi G, Benevento A, Dionigi R. Outcome and long term results of surgical resection for gastrointestinal stromal tumors (GIST). Scand J Surg 2003; 92: 195-199. [CrossRef] 